\title{
Particle transport and energization associated with disturbed magnetospheric events
}

\author{
Sorin Zaharia, C. Z. Cheng and Jay R. Johnson \\ Plasma Physics Laboratory, Princeton University, Princeton, NJ 08543
}

\begin{abstract}
Energetic particle flux enhancement events observed by satellites during strongly disturbed events in the magnetosphere (e.g., substorms, storm sudden commencements, etc.) are studied by considering interaction of particles with Earthward propagating electromagnetic pulses of westward electric field and consistent magnetic field of localized radial and azimuthal extent in a background magnetic field. The energetic particle flux enhancement is mainly due to the betatron acceleration process: particles are swept by the Earthward propagating electric field pulses via the $\mathbf{E} \times \mathbf{B}$ drift toward the Earth to higher magnetic field locations and are energized because of magnetic moment conservation. The most energized particles are those which stay in the pulse for the longest time and are swept the longest radial distance toward the Earth. Assuming a constant propagating velocity of the pulse we obtain analytical solutions of particle orbits. We examine substorm energetic particle injection by computing the particle flux and comparing with geosynchronous satellite observations. Our results show that for pulse parameters leading to consistency with observed flux values, the bulk of the injected particles arrive from distances less than $9 \mathrm{R}_{\mathrm{E}}$, which is closer to the Earth than the values obtained by the previous model [1] and is also closer to the distances obtained by the injection boundary model.
\end{abstract}




\section{INTRODUCTION}

Energetic electron and ion flux enhancement events in the magnetosphere are usually related to strongly disturbed events such as substorms and storm sudden commencements (SSC). It is well known that energetic particles appear at geosynchronous orbit $\left(6.6 \mathrm{R}_{\mathrm{E}}\right)$ subsequent to a substorm expansion phase, in a narrow "wedge" at or near local midnight [2]. The increase in the flux of these high-energy particles can be very large (two or three orders of magnitude larger than the "quiet time" flux). This increase happens simultaneously for energetic particles having a broad range of energies, and for this reason these events have been called "dispersionless injections." Similar flux increases, involving ions and electrons of much higher energies, characterize the formation of new radiation belts following storm sudden commencements (SSC). Investigations using two radially displaced satellites showed [3] that the particle injection occurs first at the outer satellite, thus suggesting that the injected particles come from farther out in the magnetosphere.

Besides their "dispersionless" nature, another feature of the energetic particle injection is the appearance of subsequent peaks at periodic time intervals after the first flux increase, these subsequent peaks manifesting greater and greater dispersion. These peaks are called "drift echoes" [4], and their explanation resides in the $\nabla \mathbf{B}$ and curvature drifts of particles, eastward for electrons and westward for ions [5].

Several models attempting to explain these injections have been proposed in the past. One promising model is based on an Earthward propagating electromagnetic field pulse: particles are energized via the betatron acceleration mechanism (based on magnetic moment conservation) as they are swept by the pulse to a location of higher magnetic field strength closer to the Earth. While test particle simulations based on this model have been extensively performed $[1,6]$, in this paper we seek greater physical insight by providing analytical solutions for non-relativistic particle motion. Our field model and particle orbit solutions are qualitatively different from the previous simulations $[1,6]$ in that by using pulse fields obtained in the cylindrical symmetry, we obtain that enhanced energetic particle fluxes ob- 
served at geosynchronous orbit during substorms are due to particles coming mainly from distances less than $9 R_{\mathrm{E}}$ away from the Earth, which is much closer to the Earth than the distance obtained by the previous simulation studies.

Past investigations [7] showed that large transient electric fields exist in the plasma sheet during the substorm expansion phase. We consider the electric field to be accompanied by a consistent magnetic field, forming a pulse that propagates towards the Earth. Correlation studies which support this picture of consistent inductive $\mathbf{E}$ and $\mathbf{B}$ fields have been previously performed [8].

\section{ENERGETIC PARTICLE FLUX ENHANCEMENT MODEL}

In order to consider the effect of the disturbed magnetospheric event on particle transport, we consider an Earthward-propagating pulse with velocity $\mathbf{V}_{\mathbf{0}}$ consisting of westward $\mathbf{E}$ and consistent $\delta \mathbf{B}$. The electric and magnetic fields of the pulse are related by Faraday's law:

$$
\frac{\partial(\delta \mathbf{B})}{\partial t}=-\nabla \times \mathbf{E}
$$

We consider the cylindrical $(r, \phi, z)$ coordinate system with $r=0$ at the center of the Earth, $z=0$ defines the equatorial plane (where our particle motion takes place), and $\phi$ is zero at midnight, positive eastward (see Fig. 1). Then, Eq. (1) in the z-direction for an azimuthally directed electric field is:

$$
\frac{\partial}{\partial r}\left(r E_{\phi}\right)=-\frac{\partial\left(r \delta B_{z}\right)}{\partial t}
$$

Assuming that the pulse propagates Earthward (in the negative $r$ direction) with a constant velocity $V_{0}$, it follows from (2) that the solution has the form $r E_{\phi}=f\left(r+V_{0} t, \phi\right)$ and

$$
\delta B_{z}=-\frac{E_{\phi}}{V_{0}}
$$

For $E_{\phi}<0$ and $V_{0}>0$ as seen in Fig. 1, we have $\delta B_{z}>0$. In our examples we will show how similar results for the particle flux can be obtained using different pulse parameters. 


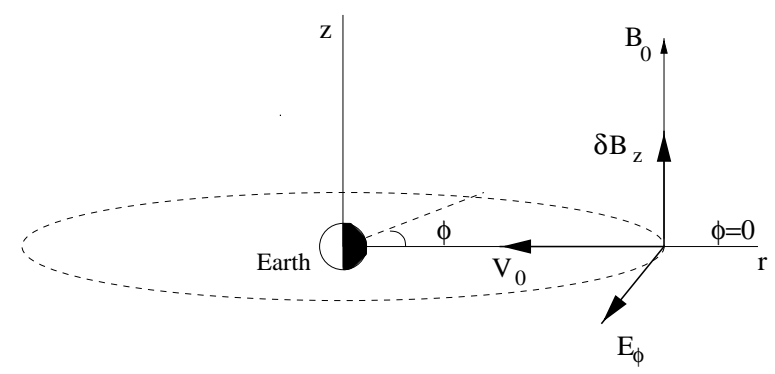

FIG. 1. Electric field $E_{\phi}$ and magnetic field $\delta B_{z}$ of the Earthward-propagating pulse; $B_{0}$ is the background magnetic field.

The particle motion will be calculated in the combined pulse and background magnetic fields. The motion conserves the magnetic moment, because the temporal variation of the pulse is much longer than the particle gyration time scale and the spatial variation of the pulse is much larger scales than the particle gyro-radii. For particle energy in the range of $100 \mathrm{keV}$ and pulse speed $\sim 200 \mathrm{~km} / \mathrm{s}$ and pulse scale length $\sim 10,000 \mathrm{~km}$, we have the following relations between gyro-periods $T_{c e} \sim 10^{-4} \mathrm{~s} \ll T_{c i} \leq 1 \mathrm{~s} \ll\left|E_{\phi} /\left(\partial E_{\phi} / \partial t\right)\right| \sim 50 \mathrm{~s}$ and gyro-radii $\rho_{e} \sim 1 \mathrm{~km} \ll \rho_{i} \sim 100 \mathrm{~km} \ll\left|E_{\phi} /\left(\partial E_{\phi} / \partial r\right)\right| \sim 10,000 \mathrm{~km}$, so the first adiabatic invariant is easily conserved by ion and electron motions in the pulse.

We only consider $90^{\circ}$ pitch angle particles with $v_{\|}=0$. Thus, the particle orbit is governed by the guiding center equation of motion:

$$
\mathbf{V}_{\mathbf{g c}}=\frac{d \mathbf{r}}{d t}=\mathbf{V}_{\mathbf{E} \times \mathbf{B}}+\mathbf{V}_{\nabla \mathbf{B}}
$$

where

$$
\begin{gathered}
\mathbf{V}_{\mathbf{E} \times \mathbf{B}}=\frac{\mathbf{E} \times \mathbf{B}}{B^{2}}, \\
\mathbf{V}_{\nabla B}=\frac{\mu}{q B^{2}} \mathbf{B} \times \nabla \mathbf{B},
\end{gathered}
$$

$q$ is the particle charge and $\mu=m v_{\perp}^{2} /(2 B)$ is its magnetic moment. Eq. (6) is the nonrelativistic form of the $\nabla \mathbf{B}$ drift. Our non-relativistic theory can be applied to ion injection during any magnetospheric event and may also be used to approximate electron motion for 
energies lower than the electron rest energy. There is no curvature drift in (4) because we only consider particles with $v_{\|}=0$.

One notices that in the moving pulse the field varies both in time and space, so one would have to include the polarization drift $\mathbf{V}_{\mathbf{p}}=m \dot{\mathbf{E}}_{\perp} /\left(q B^{2}\right)$ in Eq. (4). However, a comparison between this drift and the $\nabla \mathbf{B}$ drift which is in the same direction shows that the former is much smaller for particles with speeds above the pulse speed $V_{0}$, i.e. ions with energies above $100 \mathrm{eV}$. This is much below the average proton energy in our regions of interest, so we can neglect the polarization drift.

The variation of the particle energy as it moves in the electromagnetic fields of the pulse is then given by [9]:

$$
\frac{d W}{d t}=q \mathbf{V}_{\mathbf{g c}} \cdot \mathbf{E}+\mu \frac{\partial B}{\partial t}+\mathcal{O}\left(\epsilon^{2}\right)
$$

where $\mathcal{O}\left(\epsilon^{2}\right)$ represents terms of order $\epsilon^{2}$ and higher, with $\epsilon=\rho / L \ll 1$ ( $L$ is the scale length over which the pulse fields vary). The $\mathbf{E} \times \mathbf{B}$ drift gives no contribution to $\mathbf{V}_{\mathbf{g c}} \cdot \mathbf{E}$, so $\mathbf{V}_{\mathbf{g c}} \cdot \mathbf{E}=\mathbf{V}_{\nabla \mathbf{B}} \cdot \mathbf{E}=\mu \nabla \mathbf{B} \cdot \mathbf{V}_{\mathbf{g c}} / q$. Because $\mathbf{V}_{\mathbf{g c}}=d \mathbf{r} / d t$, Eq. (7) becomes (neglecting the higher-order terms):

$$
\frac{d W}{d t}=\mu \nabla \mathbf{B} \cdot \frac{d \mathbf{r}}{d t}+\mu \frac{\partial B}{\partial t}=\mu \frac{d B}{d t}
$$

This shows that as long as $\mu$ is conserved the particle energy is changed by changing the magnetic field intensity at its location (either by the particle moving to locations with a different magnetic field intensity or by the time variation of the field intensity). The convective term in Eq. (8) represents the change in energy by moving the particle gyrocenter to locations with different magnetic field intensity. Alternatively, from Eq. (7) it can be seen as the change in energy by moving the particle gyro-center with the $\nabla \mathbf{B}$ drift along the electric field direction. These two alternate points of view are equivalent and describe the same process, which is the change in energy due to the particle guiding center motion in the direction of the electric field, while $\mu$ is conserved. The second term in Eq. (8) is the inductive effect of the time-dependent $\mathbf{B}$-field. Together, these two terms provide the 


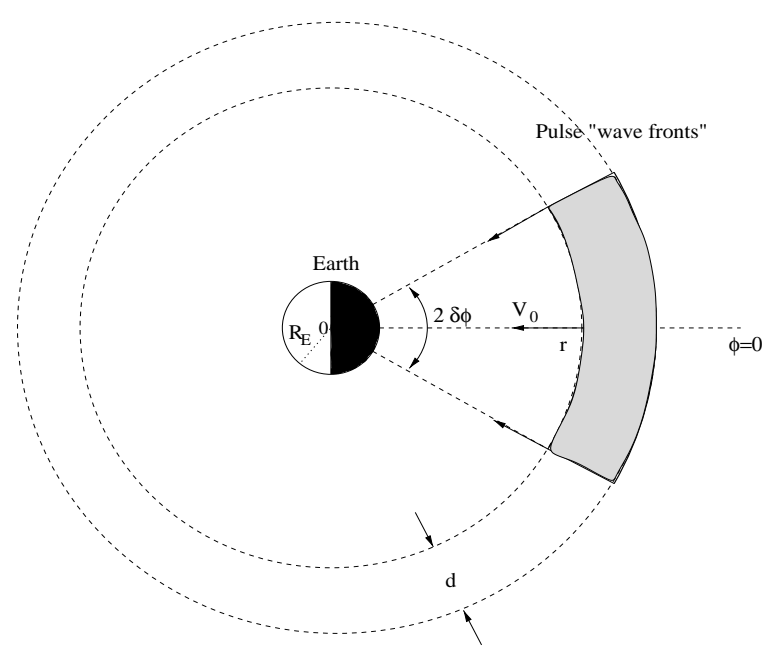

FIG. 2. Pulse geometry: the fields are non-zero inside the shaded region, which has a radial width and an azimuthal extent $2 \delta \phi$, and is centered around midnight.

adiabatic change in energy depending only on the change in the magnetic field intensity. The second term only is responsible for particle acceleration in a betatron, however in a loose way the whole process described by Eq. (8) is called "betatron acceleration."

From Eq. (8) the change in particle energy depends only on the magnetic fields at the particle initial and final positions, and the final energy of the particle is

$$
W=W_{0} \frac{B_{f}}{B_{i}}
$$

where $W_{0}$ is the particle energy at the initial position $r_{0}$ with magnetic field $B_{i} . B_{f}$ is the total magnetic field at the particle position $r$ after interacting with the pulse. If we model the background magnetic field in the equatorial plane by a dipole-like field with $\mathbf{B}_{\mathbf{0}}(r, \phi, z=0)=\mathrm{B}_{\mathrm{E}}\left(\mathrm{R}_{\mathrm{E}} / r\right)^{3} \hat{\mathbf{z}}$, where $\mathrm{B}_{\mathrm{E}}$ is a constant, then the particle energy is enhanced by a factor $W / W_{0}=\left(r_{0} / r\right)^{3}$. Note that in our model the value of $\mathrm{B}_{\mathrm{E}}$ during the particle injection phase will be adjusted (usually lower than the value of the field at the Earth's surface) to include the effect of the ring current.

With these considerations in mind, we are interested in solving Eq. (4) in a pulse having the electric field of the following form:

$$
r E_{\phi}=-\mathcal{E}_{0} \mathcal{R}\left(r+V_{0} t\right) \Phi(\phi)
$$


where $\mathcal{E}_{0}$ is a constant and has the dimension of distance times electric field, $\mathcal{R}\left(r+V_{0} t\right)$ is a radial wave form normalized to unity at the maximum amplitude, and $\Phi(\phi)=1$ inside a "wedge" centered around midnight and of finite azimuthal extent $2 \delta \phi$ (see Fig. 2), and $\Phi(\phi)=0$ elsewhere. There is no variation of the field in the $z$ direction, and particles only move in the equatorial plane $z=0$.

The radial equation of motion for guiding centers of particles moving in the pulse is

$$
\frac{d r}{d t}=\frac{-V_{0} \mathcal{E}_{0} \mathcal{R}\left(r+V_{0} t\right)}{V_{0} \mathrm{~B}_{\mathrm{E}} \mathrm{R}_{\mathrm{E}}\left(\frac{\mathrm{R}_{\mathrm{E}}}{r}\right)^{2}+\mathcal{E}_{0} \mathcal{R}\left(r+V_{0} t\right)}
$$

which can be exactly solved with the solution

$$
\frac{1}{r}-\frac{1}{r_{i}}=\frac{\mathcal{E}_{0}}{V_{0} \mathrm{~B}_{\mathrm{E}} \mathrm{R}_{\mathrm{E}}^{3}} \int_{\xi_{i}}^{\xi} \mathcal{R}(\xi) d \xi
$$

where $\xi_{i}=r_{i}+V_{0} t_{i}, \xi=r+V_{0} t, r_{i}$ is the initial particle position at the time $t_{i}$ and $r$ is the particle position at the time $t$ in the pulse. We note that the solution for $r(t)$ depends only on the radial electric field wave form $\mathcal{R}\left(r+V_{0} t\right)$ and does not depend on the azimuthal position as long as particles stay inside the pulse. The equation of the particle azimuthal motion also depends on the radial wave form of the pulse. To solve for $\phi$ it is easier to use $r$ as the dependent variable instead of $t$ and the azimuthal equation of motion is given by

$$
\frac{d \phi}{d r}=\frac{\mu}{q \mathcal{E}_{0}}\left(\frac{3 \mathrm{~B}_{\mathrm{E}} \mathrm{R}_{\mathrm{E}}^{3}}{\mathcal{R} r^{4}}+\frac{\mathcal{E}_{0}}{V_{0} r^{2}}-\frac{\mathcal{E}_{0}}{V_{0} r \mathcal{R}} \frac{\partial \mathcal{R}}{\partial r}\right)
$$

In the following sections we apply this expression and obtain the exact solutions of the particle orbit by choosing a radial trapezoidal wave form $\mathcal{R}$ as shown in Fig. 3 . The radial pulse form at the time the particle encounters the pulse is shown in Fig. 3 with $\mathcal{R}=\left(\xi-\xi_{0}\right) / \Delta_{1}$ for $\xi_{0}=r_{0}+V_{0} t_{0} \leq \xi \leq \xi_{1}=\xi_{0}+\Delta_{1}, \mathcal{R}=1$ for $\xi_{1} \leq \xi \leq \xi_{2}=\xi_{1}+\Delta_{2}$, $\mathcal{R}=1-\left(\xi-\xi_{2}\right) / \Delta_{3}$ for $\xi_{2} \leq \xi \leq \xi_{3}=\xi_{2}+\Delta_{3}$, and $\mathcal{R}=0$ otherwise. We solve for the particle radial and azimuthal motion in the three sections of the trapezoidal pulse. While our pulse form is a simplified one, the results are quite general: an arbitrary wave form can be approximated by a number of pulses of trapezoidal form, and this number and the width 


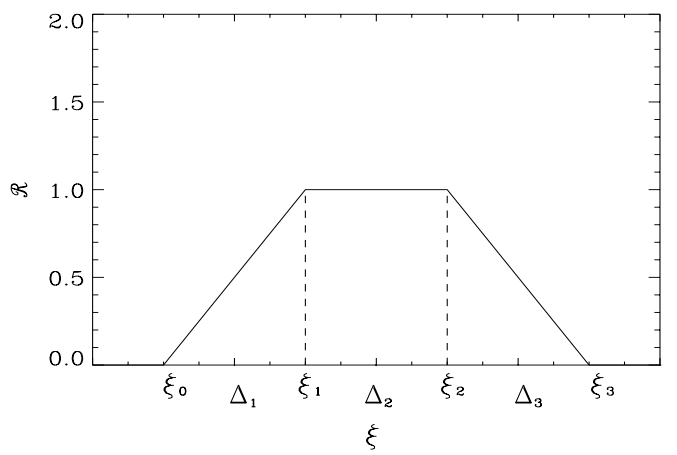

FIG. 3. The radial pulse form of $\mathcal{R}$ at $t=t_{0}$.

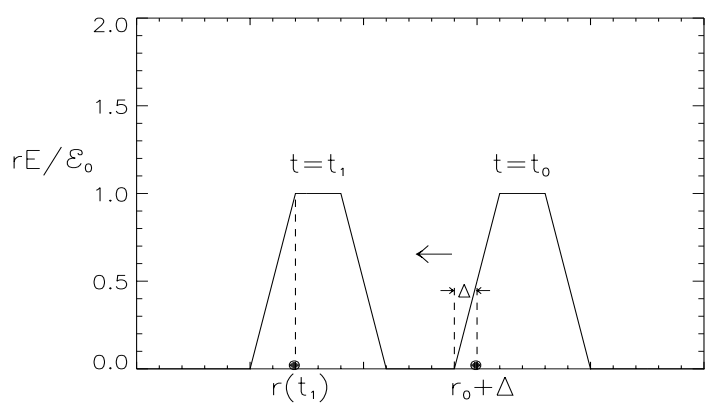

FIG. 4. Particle that enters at $r_{0}+\Delta$ at time $t_{0}$ and exits radially from the first part of the pulse, at $t=t_{1}$.

of the individual pulses can be adjusted to accurately represent the original wave form. The azimuthal motion in any of these pulses will be of one of the three kinds encountered in our trapezoidal pulse.

There are four classes of particles that interact with the pulse: the first is particles that enter and leave the pulse radially; the second is particles that enter the pulse radially but leave azimuthally; the third is particles that enter the pulse azimuthally but leave radially; and the fourth is particles that enter and leave the pulse azimuthally. The orbit solutions for these particles can be obtained analytically. In the following we present the particle orbit solutions in each of the three regions of the pulse. 


\section{A. Motion in the first part of the pulse}

Considering particles that enter the first part of the pulse at $t=t_{0}$ at initial position $\left(r_{i}=r_{0}+\Delta, \phi_{i}\right)$ where $0<\Delta<\Delta_{1}$ and $\left|\phi_{i}\right| \leq \delta \phi$, their orbit solutions can be obtained exactly. From Eq. (11) the solution for $r$ is

$$
\frac{2 V_{0} \mathrm{~B}_{\mathrm{E}} \mathrm{R}_{\mathrm{E}}^{3}}{\mathcal{E}_{0} \Delta_{1}}\left(\frac{1}{r}-\frac{1}{r_{i}}\right)+\left(\frac{\Delta}{\Delta_{1}}\right)^{2}=\left[\frac{r-r_{0}+V_{0}\left(t-t_{0}\right)}{\Delta_{1}}\right]^{2}
$$

and the solution for $\phi$ can be obtained by integrating Eq. (13) and is given by

$$
\begin{aligned}
\phi-\phi_{i} & =\frac{-6 \mu}{q}\left(\frac{\mathrm{B}_{\mathrm{E}} \mathrm{R}_{\mathrm{E}}^{3} \Delta_{1}}{2 V_{0} \mathcal{E}_{0}}\right)^{1 / 2}\left\{\frac{1}{5}\left[\left(\frac{1}{r}-\frac{1}{a}\right)^{5 / 2}-\left(\frac{1}{r_{i}}-\frac{1}{a}\right)^{5 / 2}\right]\right. \\
& \left.+\frac{2}{3 a}\left[\left(\frac{1}{r}-\frac{1}{a}\right)^{3 / 2}-\left(\frac{1}{r_{i}}-\frac{1}{a}\right)^{3 / 2}\right]+\frac{1}{a^{2}}\left[\left(\frac{1}{r}-\frac{1}{a}\right)^{1 / 2}-\left(\frac{1}{r_{i}}-\frac{1}{a}\right)^{1 / 2}\right]\right\} \\
+ & \frac{\mu}{q V_{0}}\left\{\left(\frac{1}{r_{i}}-\frac{1}{r}\right)+\left(\frac{\mathcal{E}_{0} r_{0}}{2 V_{0} \mathrm{~B}_{\mathrm{E}} \mathrm{R}_{\mathrm{E}}^{3} \Delta_{1}}\right)^{1 / 2}\left[\sin ^{-1}\left(\frac{2 r_{i}}{a}-1\right)-\sin ^{-1}\left(\frac{2 r}{a}-1\right)\right]\right\}
\end{aligned}
$$

where $1 / a=1 / r_{i}-\left(\Delta / G_{1} \Delta_{1}\right)^{2}$ and $G_{1}^{2}=2 V_{0} \mathrm{~B}_{\mathrm{E}} \mathrm{R}_{\mathrm{E}}^{3} /\left(\mathcal{E}_{0} \Delta_{1} r_{0}\right)$. If particles enter the pulse radially, $\Delta=0$ and $r_{i}=r_{0}$. If particles enter the pulse azimuthally, $\phi_{i}=\delta \phi$ for ions and $\phi_{i}=-\delta \phi$ for electrons.

Particles can leave the pulse azimuthally (if $|\phi|$ as given by (15) is larger than $\delta \phi$ ), or otherwise exit radially at

$$
r\left(t_{1}\right)=\frac{r_{i}}{1+\frac{\mathcal{E}_{0}\left(\Delta_{1}^{2}-\Delta^{2}\right) r_{i}}{2 V_{0} \Delta_{1} \mathrm{~B}_{\mathrm{E}} \mathrm{R}_{\mathrm{E}}^{3}}}
$$

where $t_{1}$ is the time when particles exit the first part of the pulse radially and is given by

$$
t_{1}-t_{0}=\left[\Delta_{1}-\Delta+r_{i}-r\left(t_{1}\right)\right] / V_{0}
$$

It is interesting to note that if the gradient of the pulse magnetic field is larger than the gradient of the background field so that the net magnetic field gradient is radially outward, ions will drift eastward with $\phi$ increasing as $r$ decreases as shown in Fig. 5. For such a case the first term in Eq. (7) will cause the ions to lose energy because they move against the 


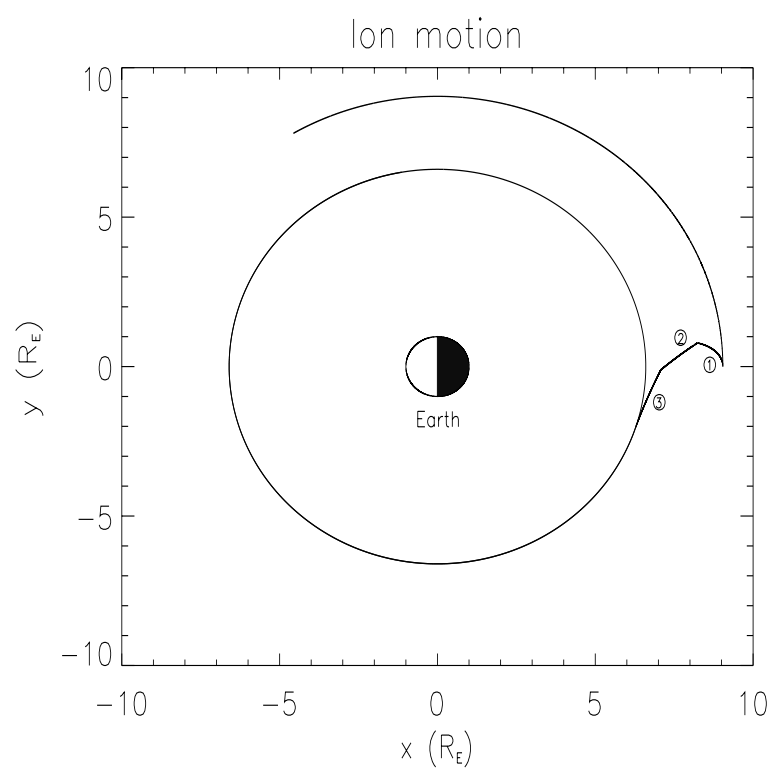

FIG. 5. Motion of an equatorially-trapped ion in the pulse; the initial energy of the ion is 58 $\mathrm{keV}$; the ion initially drifts azimuthally at $9 \mathrm{R}_{\mathrm{E}}$, until it meets the pulse and enters it radially at $\phi=0$ (midnight); the ion subsequently stays in all 3 parts of the pulse, until it goes out of the pulse also radially, at $6.6 \mathrm{R}_{\mathrm{E}}$, and then drifts around the Earth at that distance; the different motions in the 3 parts are numbered accordingly; the final ion energy is $150 \mathrm{keV}$; the pulse parameters are: $\mathcal{E}_{0}=7 \mathrm{mV} / \mathrm{m} \cdot 9 \mathrm{R}_{\mathrm{E}}, 2 \delta \phi=30^{\circ}, V_{0}=200 \mathrm{~km} / \mathrm{s}, \Delta_{1}=\Delta_{2}=\Delta_{3}=9,000 \mathrm{~km}$

electric field (similar argument applies for electrons). However, the second term $\mu \partial B / \partial t$ in Eq. (7) is usually positive and larger in magnitude than the first term and thus particles will move to a higher magnetic field location and gain energy.

\section{B. Motion in the second part of the pulse}

For particles entering the second part of the pulse at time $t_{i}$ and position $\left(r_{i}, \phi_{i}\right)$ where $r_{i}=r_{1}+\Delta_{2}-\Delta, 0 \leq \Delta \leq \Delta_{2}$, and $\left|\phi_{i}\right| \leq \delta \phi$, the radial solution is obtained from Eq. (11) and is given by

$$
\frac{1}{r}-\frac{1}{r_{i}}=\frac{\mathcal{E}_{0}}{\mathrm{~B}_{\mathrm{E}} \mathrm{R}_{\mathrm{E}}^{3}}\left[r-r_{i}+V_{0}\left(t-t_{i}\right)\right]
$$




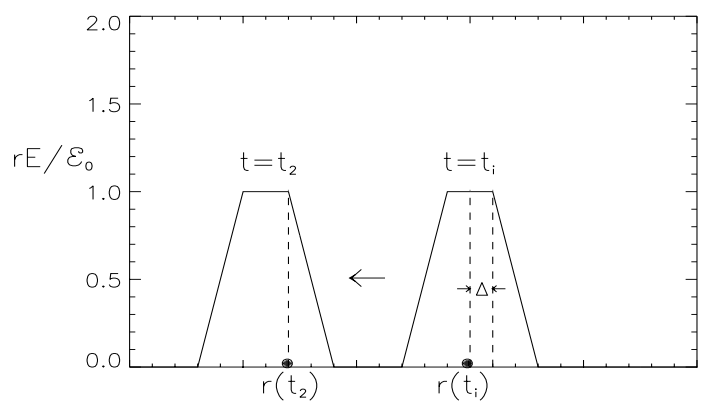

FIG. 6. Particle that enters the second part of the pulse at $r_{i}=r_{1}+\Delta_{2}-\Delta$ and time $t_{i}$ and exits radially at time $t_{2}$

and the azimuthal solution is given by

$$
\phi-\phi_{i}=\frac{\mu}{q}\left[\frac{\mathrm{B}_{\mathrm{E}} \mathrm{R}_{\mathrm{E}}^{3}}{\mathcal{E}_{0}}\left(\frac{1}{r_{i}^{3}}-\frac{1}{r^{3}}\right)+\frac{1}{V_{0}}\left(\frac{1}{r_{i}}-\frac{1}{r}\right)\right]
$$

Particles which exit this second part radially do so at $r_{2}=r\left(t_{2}\right)=r_{i}-V_{0}\left(t_{2}-t_{i}\right)+\Delta$ at time $t_{2}$, and $r_{2}$ is obtained from Eq. (12) and is given by

$$
r_{2}=\frac{r_{i}}{1+\frac{\mathcal{E}_{0} \Delta r_{i}}{V_{0} \mathrm{~B}_{\mathrm{E}} \mathrm{R}_{\mathrm{E}}^{3}}}
$$

If particles enter this second part radially at $t_{i}=t_{1}$, then $r_{i}=r_{1}$ and $\Delta=\Delta_{2}$ in the above orbit solutions. The radial positions for particles that enter and exit the second part of the pulse are shown in Fig. 6. The azimuthal drift is mostly given by the background magnetic field (the gradient of the pulse field is smaller), and ions drift westward (see Fig. 5) and electrons eastward.

\section{Motion in the third part of the pulse}

The equations of motion in the third part of the pulse (with decreasing field) are similar to the ones in the first part. For particles entering this third part at time $t_{i}$ and position $\left(r_{i}, \phi_{i}\right)$ where $r_{i}=r_{2}+\Delta_{3}-\Delta, 0 \leq \Delta \leq \Delta_{3}$, and $\left|\phi_{i}\right| \leq \delta \phi$, the radial solution of Eq. (11) is 


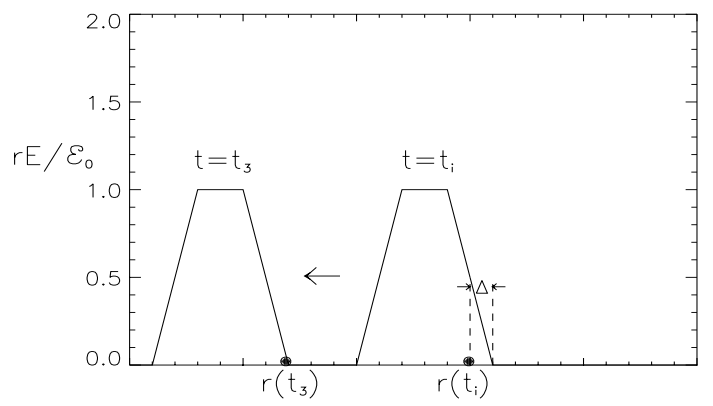

FIG. 7. Particle that enters the third part of the pulse at $r_{i}=r_{2}+\Delta_{3}-\Delta$ and time $t_{i}$ and exits radially at time $t_{3}$

$$
\frac{1}{r}-\frac{1}{r_{i}}=\frac{\mathcal{E}_{0} \Delta_{3}}{2 V_{0} \mathrm{~B}_{\mathrm{E}} \mathrm{R}_{\mathrm{E}}^{3}}\left[\left(\frac{\Delta}{\Delta_{3}}\right)^{2}-\left(\frac{r-r_{i}+V_{0}\left(t-t_{i}\right)}{\Delta_{3}}\right)^{2}\right]
$$

and the azimuthal solution is obtained by integrating Eq. (13) and is given by

$$
\begin{aligned}
\phi-\phi_{i} & =\frac{6 \mu}{q}\left(\frac{\mathrm{B}_{\mathrm{E}} \mathrm{R}_{\mathrm{E}}^{3} \Delta_{3}}{2 V_{0} \mathcal{E}_{0}}\right)^{1 / 2}\left\{\frac{1}{5}\left[\left(\frac{1}{b}-\frac{1}{r}\right)^{5 / 2}-\left(\frac{1}{b}-\frac{1}{r_{i}}\right)^{5 / 2}\right]\right. \\
& \left.-\frac{2}{3 b}\left[\left(\frac{1}{b}-\frac{1}{r}\right)^{3 / 2}-\left(\frac{1}{b}-\frac{1}{r_{i}}\right)^{3 / 2}\right]+\frac{1}{b^{2}}\left[\left(\frac{1}{b}-\frac{1}{r}\right)^{1 / 2}-\left(\frac{1}{b}-\frac{1}{r_{i}}\right)^{1 / 2}\right]\right\} \\
& +\frac{\mu}{q V_{0}}\left(\frac{1}{r_{i}}-\frac{1}{r}\right)+\frac{\mu \sqrt{b}}{q V_{0} G_{3} \Delta_{3}} \ln \frac{2 r-b+\sqrt{(2 r-b)^{2}-b^{2}}}{2 r_{i}-b+\sqrt{\left(2 r_{i}-b\right)^{2}-b^{2}}}
\end{aligned}
$$

where $G_{3}^{2}=2 V_{0} \mathrm{~B}_{\mathrm{E}} \mathrm{R}_{\mathrm{E}}^{3} / \mathcal{E}_{0} \Delta_{3}$ and $1 / b=\Delta^{2} / \Delta_{3}^{2} G_{3}^{2}+1 / r_{i}$. For particles exiting from the pulse radially (as shown in Fig. 7) at $r_{3}=r\left(t_{3}\right)=r_{i}+\Delta-V_{0}\left(t_{3}-t_{i}\right)$, we obtain from Eq. (12)

$$
r_{3}=\frac{r_{i}}{1+\frac{\mathcal{E}_{0} \Delta r_{i}}{2 V_{0} \mathrm{~B}_{\mathrm{E}} \mathrm{R}_{\mathrm{E}}^{3}}}
$$

For particles entering the third part radially, the solutions are obtained by setting $r_{i}=r_{2}$, $\Delta=\Delta_{3}$, and $t_{i}=t_{2}$. In Fig. 5 one can see that in the third part of the pulse the background field and pulse field gradients give rise to drifts in the same azimuthal direction, and so the westward (for ions) drift is more pronounced than in the second part. 


\section{General discussion of particle orbits}

For particles that enter the first part of the pulse radially and do not exit azimuthally from the pulse, the final radial exit position $r_{3}$ is related to the initial radial distance $r_{0}$ only through the radial integration of the entire electric field structure as shown in Eq. (12). For a trapezoidal pulse the integration can be easily performed and we can express the initial coordinate $r_{0}$ as a function of $r_{3}$ :

$$
r_{0}=\frac{r_{3}}{1-\frac{\mathcal{E}_{0}\left(\Delta_{1}+2 \Delta_{2}+\Delta_{3}\right) r_{3}}{2 V_{0} B_{\mathrm{E}} \mathrm{R}_{\mathrm{E}}^{3}}}
$$

where the sum $\Delta_{1}+2 \Delta_{2}+\Delta_{3}$ is just the value of the electric field integral $\int_{\xi_{i}}^{\xi_{f}} \mathcal{R}(\xi) d \xi$ for the trapezoidal pulse. Thus, the initial particle radial distance is related to the final particle radial distance through the ratio of this electric field integral to the pulse velocity $V_{0}$. For a general pulse form (as shown in Fig. 8) the electric field integral can be approximated by a finite-difference integration of the shaded area delimited by the curve representing $r E$ between the points of entrance and exit from the pulse. This integral is equivalent to a number of trapezoidal pulses. The generality of the solution makes it straightforward to calculate the particle motion in other situations such as a train of pulses or when a reflected pulse is present, etc.

It is to be noted that if $\Delta_{1}$ and $\Delta_{3}$ of the trapezoidal pulse are too small, the large gradients in the first and final part of the pulse will eventually cause the particles to exit the pulse azimuthally. Fig. 9 shows the variation of the final radial distance $r_{3}$ versus the initial distance $r_{0}$, and we see that for any set of pulse parameters, there is a maximum final distance to which particles that stay all the time in the pulse can be swept - this distance is the limit of $r_{3}$ given by (24) as $r_{0} \rightarrow \infty$. This limiting value is

$$
r_{3 \max }=\frac{2 V_{0} \mathrm{~B}_{\mathrm{E}} \mathrm{R}_{\mathrm{E}}^{3}}{\mathcal{E}_{0}\left(\Delta_{1}+2 \Delta_{2}+\Delta_{3}\right)}
$$

There are different kinds of particle orbits (as defined by how they enter/exit from the pulse). Particles that enter and exit the pulse radially are the ones that will travel the largest 


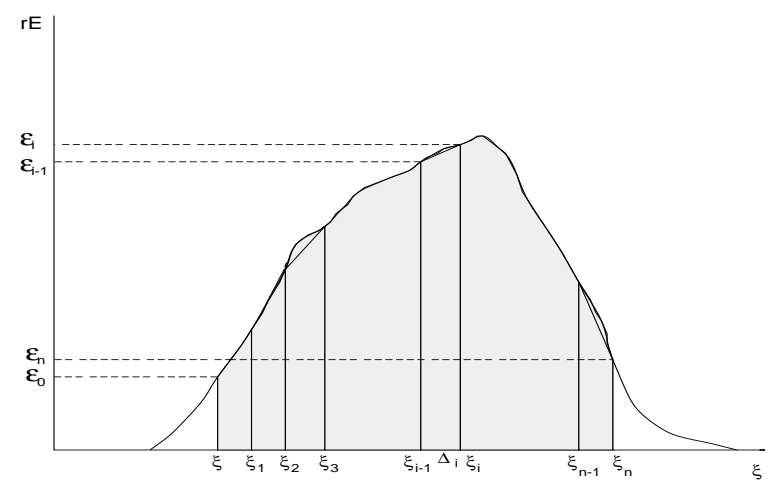

FIG. 8. An arbitrary pulse form can be de-composed into a large number of trapezoidal pulses, the motion in which has been completely solved.

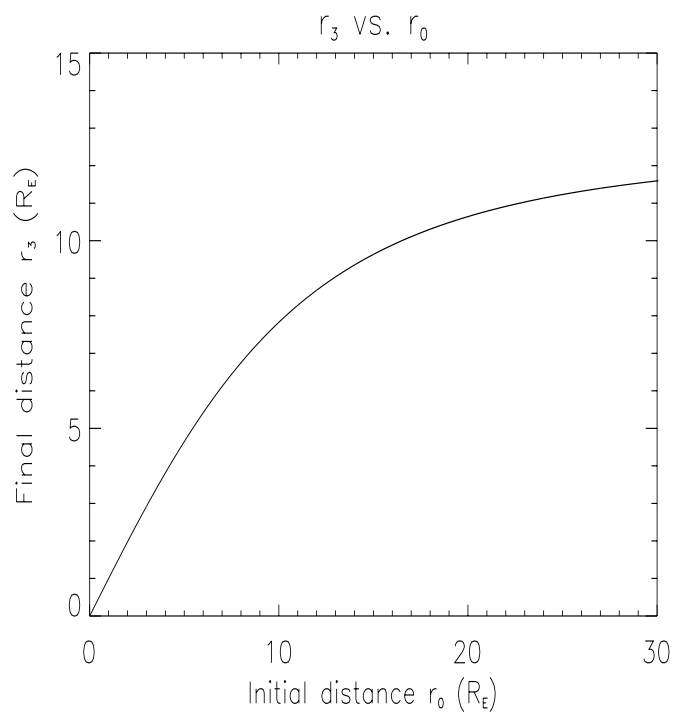

FIG. 9. Final versus initial radial distance for particles that stay in all three parts of the pulse (with the starting position of the pulse taken at a distance greater than $r_{0}$ ); the pulse parameters are the same as in Fig. 5. 
distance and have their energy enhanced by the largest factor. Because the background particle distribution decreases with both energy and radial distance, the energetic particle flux enhancement at the observation point will be mainly due to these particles. Particles that enter or exit the pulse azimuthally contribute less to the enhanced particle fluxes.

We now take a closer look at the azimuthal motion. We discuss ions only, and the electron case is similar except for the different drift direction. In parts 2 and 3 of the pulse ions drift westward, but in the first part they can drift either eastward or westward depending on whether or not the gradient of the pulse magnetic field is larger in magnitude than the gradient of the background field. A case where the azimuthal drift is entirely eastward is that of particle 1 in Fig. 10, while particle 3, starting at a closer distance, encounters a much stronger background magnetic field (and gradient), and its azimuthal drift is westward. It is possible for an ion to arrive at the distance where the background and pulse field gradients are equal but opposite while the ion is still in the first part of the pulse, in which case it will reverse its azimuthal motion from an eastward direction to a westward one (particle 2 in Fig. 10). For this case the ion will be able to stay even in a very narrow pulse, because the net azimuthal drift is much lower than in the other two cases.

The condition for particles not to exit azimuthally from the pulse is $|\phi| \leq \delta \phi$, which gives for each initial angle the maximum $\mu$ particles entering the pulse may have in order to stay in all three parts of the pulse. The value of $\mu_{\max }$ can be obtained from Eq. (15), (19) and (22). Higher energy particles with $\mu>\mu_{\max }$ will exit the pulse azimuthally. These particles, in order to be swept to the same location as the particles exiting the pulse radially, must come from initial distances closer to the Earth, and their energy enhancement will be smaller.

Because the number of particles that enter the pulse azimuthally is usually small for fast pulses, their contribution to the flux increase will not be large; however, they can account for the initial flux increase. Entering the first part of the pulse is only possible at distances for which the gradient of the pulse field is smaller than the background field gradient. As particles enter the pulse azimuthally they experience a sudden transition from zero to non- 


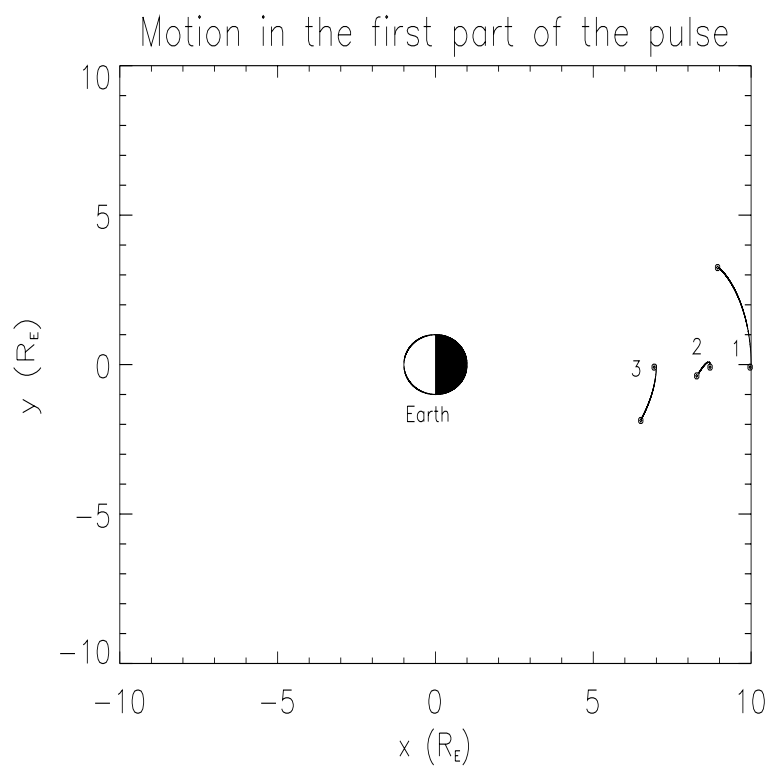

FIG. 10. Different kinds of azimuthal motion in the first part of the pulse; particles 1,2 and 3 start moving into the first part of the pulse, having the same $\phi_{i n}=0$ and the same initial energy $W=500 \mathrm{keV}$, but from different initial radial positions: $10,8.7$ and $7 \mathrm{R}_{\mathrm{E}}$, respectively; particle 1 drifts entirely eastward, particle 2 starts drifting eastward then reverses its drift as it arrives in regions of higher background field, and particle 3 has an entirely westward drift; the pulse parameters are $\mathcal{E}_{0}=3 \mathrm{mV} / \mathrm{m} \cdot 10 \mathrm{R}_{\mathrm{E}}, 2 \delta \phi=50^{\circ}, V_{0}=200 \mathrm{~km} / \mathrm{s}, \Delta_{1}=\Delta_{2}=\Delta_{3}=9,000 \mathrm{~km}$; the pulse starts at $10 \mathrm{R}_{\mathrm{E}}$.

zero pulse fields in the $\phi$ direction at the azimuthal limits of the pulse. The particle drift due to that gradient is ignored because there is no physical "shock" at the azimuthal edges of the pulse. Particles that cannot azimuthally enter the first portion of the pulse will stay at $|\phi|=\delta \phi$ until they enter the pulse in the second part.

\section{E. Particle flux}

Having derived the analytical orbit solutions, we now want to obtain the particle flux at the observation point (geo-synchronous orbit usually), in order to compare it with the flux measured by satellites. Some satellites measure the omnidirectional flux [10], which is the flux that covers all solid angles. Because we only consider $90^{\circ}$ pitch-angle particles, in 
order to make a meaningful comparison between theory and observation we will calculate the directional flux which is the number of particles per unit time, energy, area and solid angle coming from a given direction: $j=\partial^{2} J /(\partial \Omega \partial W)[10]$, where $J$ is the omnidirectional flux, i.e. the number of particles per unit time and area. In our case, the directional flux for $90^{\circ}$ pitch-angle particles is $j_{\perp}[10]$. For an isotropic flux, $J=4 \pi j$, but the magnetospheric particle population is not isotropic and furthermore particles with non-zero $v_{\|}$will move differently in the pulse compared to the particles we study, so we can not infer the omnidirectional flux from our model. However, we can obtain the ratio of injected flux for particles with energy within a certain energy range to the background flux in our model and compare it with the relative level of the injected omnidirectional flux to the background omnidirectional flux obtained from satellite observations. Some satellites measure the directional flux though, and in this case the problem is simpler: the comparison can be made directly between our calculated and observed fluxes.

The directional flux is related to the particle distribution function by [11]:

$$
F(\mathbf{r}, \mathbf{v}, t)=\frac{m^{2}}{2 W} j
$$

Liouville's theorem states that the particle density in phase space is conserved along the trajectory, which can be written as $d F / d t=0$ if we neglect collisions on the short time scale of the injection. So $F\left(\mathbf{r}_{i}, \mathbf{v}_{i}, t_{i}\right)=F\left(\mathbf{r}_{f}, \mathbf{v}_{f}, t_{f}\right)$. Then, from Eq. (26) $j / W$ is constant along the particle trajectory. Because the particle energy $W$ at $\mathbf{r}_{\mathbf{f}}$ is related to its initial energy at $\mathbf{r}_{\mathbf{i}}$ by the ratio of the magnetic field intensity at these positions, the differential flux can be obtained in each region of space.

To use the invariance of the phase space density along the particle trajectory, we transform the distribution function from a function of velocity to a function of energy: $F(\mathbf{v}, \mathbf{r})=1 /(4 \pi) \sqrt{m /(2 W)} f(W, r, \phi)$, so the directional flux $j$ at $r$ for a particle with energy $W$ can be expressed as

$$
j=\frac{W}{\pi \sqrt{W_{i}}}\left(\frac{1}{2 m}\right)^{3 / 2} f\left(W_{i}, r_{i}, \phi_{i}, t_{i}\right)
$$


where $W=W_{i} B(r, \phi, t) / B\left(r_{i}, \phi_{i}, t_{i}\right)$. For particles outside the pulse in a dipole-like background field $W=W_{i}\left(r_{i} / r\right)^{3}$. The above formula is valid for any position along the trajectory of a particle that starts at $\left(r_{i}, \phi_{i}\right)$ with energy $W_{i}$. One notices that the injected flux varies as $r^{-3}[11]$ and it is to be added to the background particle flux of the same energy at the injection position.

\section{PARTICLE INJECTION IN A SUBSTORM EVENT}

The solutions obtained in the previous section are restricted to non-relativistic particles, which is correct for ions with enhanced flux observed during strongly disturbed magnetospheric events and is also a good approximation for electrons with energy on the order of $100 \mathrm{keV}$ range observed during substorm injections.

\section{A. Ion injection}

We first present an application of our analytical model to an energetic proton injection event observed by LANL geosynchronous satellite sensors on January 10, 1997 (see the left panel in Fig. 11) during a substorm. For this event, we consider a three-part trapezoidal pulse of the form shown in Fig. 3, centered at midnight local time $(\phi=0)$ with the pulse parameters: $\mathcal{E}_{0}=7.5 \mathrm{mV} / \mathrm{m} \cdot 9 \mathrm{R}_{\mathrm{E}}, \Delta_{1}=\Delta_{2}=\Delta_{3}=6,000 \mathrm{~km}$, and $2 \delta \phi=30^{\circ}$. The pulse Earthward propagating speed is chosen to be $V_{0}=200 \mathrm{~km} / \mathrm{s}$. The pulse electric field will produce a particle $\mathbf{E} \times \mathbf{B}$ speed between 60 and $100 \mathrm{~km} / \mathrm{s}$ for $\mathrm{B}_{\mathrm{E}}=0.22 \mathrm{G}$, which is consistent with the observed plasma flow speed in the region between $6.6 \mathrm{R}_{\mathrm{E}}$ and $9 \mathrm{R}_{\mathrm{E}}$.

With the above parameters (which a posteriori are found to give flux levels consistent with those observed), the maximum initial radial distance $r_{i}$ of protons that can be swept by the pulse to the geosynchronous orbit $\left(r_{\text {geo }}=6.6 \mathrm{R}_{\mathrm{E}}\right)$ is calculated from Eq. (24) to be 8.6 $\mathrm{R}_{\mathrm{E}}$ so the front edge of the pulse must be at a radial distance greater than or equal to 8.6 $\mathrm{R}_{\mathrm{E}}$ in order to sweep these particles to the geosynchronous orbit. Because the pulse spans about $3 \mathrm{R}_{\mathrm{E}}$, the substorm formation region (the center of the pulse) will be at a distance 
greater than about $10 \mathrm{R}_{\mathrm{E}}$. The maximum increase in the particle energy due to betatron acceleration is about $\left(r_{i} / r_{\text {geo }}\right)^{3} \approx 2.2$ times. If $t=0$ is the moment the pulse arrives at 8.6 $\mathrm{R}_{\mathrm{E}}$, the pulse will arrive at $6.6 \mathrm{R}_{\mathrm{E}}$ at $t \approx 1 \mathrm{~min}$. Protons with initial distances very close to $6.6 \mathrm{R}_{\mathrm{E}}$ will arrive at the geosynchronous orbit first. Protons from $8.6 \mathrm{R}_{\mathrm{E}}$ are swept to the geosynchronous orbit at about $t_{3} \approx 2 \mathrm{~min} 30 \mathrm{~s}$ and these particles exit from the tail edge of the pulse. This means that the initial injection event will span a time interval of less than 2 min. During this short time interval, protons with a wide range of energies will be swept to geosynchronous orbit, thus accounting for the "dispersionless" nature of the injection. One should keep in mind that these protons arrive at geosynchronous orbit with different azimuths, and they subsequently drift around the Earth to the observing satellite location.

To find the energy range for which protons will not exit the pulse azimuthally, we compare $\phi$ obtained from Eqs. (15), (19), (22) to the pulse azimuthal limits. For our pulse parameters, the "maximum energy" for such protons at $r_{1}$ and $\phi=\delta \phi$ is $W_{1 \max } \approx 90 \mathrm{keV}$, so the maximum final energy these protons can have at its final position is $W_{1 \max } \cdot\left(r_{1} / r_{\text {geo }}\right)^{3} \approx$ $160 \mathrm{keV}$. Thus, higher energy protons will have to be swept from a distance closer to the Earth in order to arrive at $6.6 \mathrm{R}_{\mathrm{E}}$.

Now we want to obtain the injected flux. We model the initial background proton distribution to be uniform azimuthally and as a kappa distribution in energy [12]:

$$
f(W, r)=f_{0} \cdot g(r)\left[1+\frac{W}{(\kappa-1.5) \varepsilon}\right]^{-\kappa-1}
$$

with $\varepsilon$ being the average energy, $\kappa$ a parameter and $f_{0}$ a constant. To model a moderately active plasma sheet we choose $\kappa=6$ and $\varepsilon=7 \mathrm{keV}$ for protons in our region of interest $\left(6.6 \mathrm{R}_{\mathrm{E}}\right.$ to $9 \mathrm{R}_{\mathrm{E}}$ ), similar to values reported in [13] and also used in [14]. The radial dependence $g(r)$ is taken to be the one used in [1], $g(r)=\left(r / \mathrm{R}_{\mathrm{E}}-3\right)^{4} /\left(r / \mathrm{R}_{\mathrm{E}}\right)^{10}$ for $r<12 \mathrm{R}_{\mathrm{E}}$.

In order to obtain the proton flux as a function of energy and time at the observing satellite location, we need to obtain the proton flux at $\left(r_{g e o},-\delta \phi\right)$ location as a function of energy and time while the pulse moves across the geosynchronous orbit and at $\left(r_{g e o},|\phi| \leq \delta \phi\right)$ locations when the tail edge of the pulse just leaves the geosynchronous orbit. These particles 
will then $\nabla \mathbf{B}$ drift around the Earth to the observing location. We perform numerical calculations by employing our analytical orbit solutions to a distribution of particles in the region of interest. We take particle cells every $1000 \mathrm{~km}$ between $6 \mathrm{R}_{\mathrm{E}}$ and $9 \mathrm{R}_{\mathrm{E}}$, every $1.5^{\circ}$ in azimuth and with 20 different energy channels within the energy range of interest. The cells have to be spaced densely enough so that the motion of phase space points from the same cell is not chaotic, i.e. a slight variation in the initial location and energy does not influence much the final values [14]. Each particle cell is "labeled" by its initial radial distance and energy, and Eq. (27) is used to obtain the particle differential flux corresponding to that cell at the observation point. After that, we integrate the flux over the energy range of interest. We follow the motion of the cells during the injection event and the subsequent drift echoes, recording the cells that are injected at the radial region $\left[r_{\text {geo }}-\delta r, r_{g e o}+\delta r\right]$, with $\delta r=500 \mathrm{~km}$, and calculating the corresponding flux at the satellite (the geosynchronous motion of the satellite is also included in the calculation). We obtain an integrated flux over the energy range of interest, and we plot this flux with a time step of $100 \mathrm{~s}$. After we obtain the particle flux at the observing satellite location, we multiply the particle flux by an exponentially decaying function in time with an e-folding time for protons of 40 minutes for our energy ranges. This modification of particle flux after the particles are swept to the geosynchronous location is to simulate the subsequent loss (mainly by pitch-angle diffusion due to cyclotron instabilities, as shown in [15]). This practice was also used in previous calculations [1]. We also note that in our model particles interact with the pulse only once because the drift period at $r_{\text {geo }}$ is about $11 \mathrm{~min}$ for $600 \mathrm{keV}$ particles and is much larger than the time (about $2 \mathrm{~min}$ ) needed for all three parts of the pulse to pass through the geosynchronous orbit.

The simulation result is presented in Fig. 11. We notice that the flux enhancement is quite large, almost two orders of magnitude larger than the background flux in the same energy range. The small-scale fluctuations observed in later drift echoes are due to the finite number of cells considered and the time step used for plotting and become smaller as the cell number is increased. From the comparison of the simulated particle fluxes with the observed 


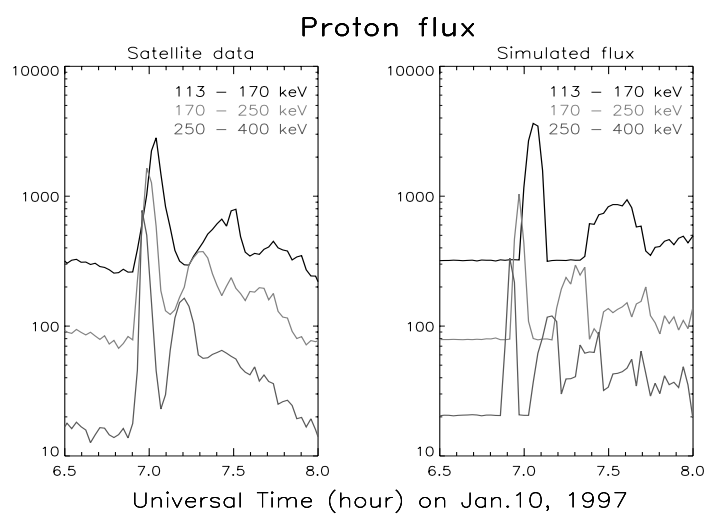

FIG. 11. Proton flux at geosynchoronous orbit, integrated over the following energy ranges, from top to bottom: $[113,170] \mathrm{keV},[170,250] \mathrm{keV},[250,400] \mathrm{keV}$; on the left is the flux measured by sensors on LANL satellite 1991-080 (at local time LT=UT+4:40); the right graph shows the simulated flux at the satellite position; both the satellite data and simulated flux plots have a time resolution of $100 \mathrm{~s}$.

ones we find a very good agreement in the major features of the particle flux even for such a simple pulse form. These features include the ratio of the injected flux versus the background flux and the relative positions of the drift echoes. There are also differences between theory and observation mainly because our model only treats $90^{\circ}$ pitch-angle particles, while the satellite sensors register all particles. Another cause for the difference is that our pulse form is a simplified one. Another difference, the higher flux between two adjacent drift echoes in the observed flux, can be due to a physical mechanism that is not included in our model; i.e., the higher-energy particles (which arrive earlier) through other processes can modify the background population and thus increasing the flux for lower energy ranges [5].

In order to explain the very large increase in the flux of particles with energy greater than $300 \mathrm{keV}$ observed in some events, our pulse parameters have to be modified, to have a larger E field and/or azimuthal extent. For example, if the azimuthal extent of the pulse is taken to be $2 \delta \phi=100^{\circ}$ with the other parameters unchanged, particles can be swept from $8.6 \mathrm{R}_{\mathrm{E}}$ with energies reaching $530 \mathrm{keV}$ at $6.6 \mathrm{R}_{\mathrm{E}}$ because particles with higher initial energy can stay in the pulse and be swept to the geosynchronous location. The flux increase depends even more sharply on the E-field of the pulse. A field of $15 \mathrm{mV} / \mathrm{m}$ at $9 R_{\mathrm{E}}$ (with 


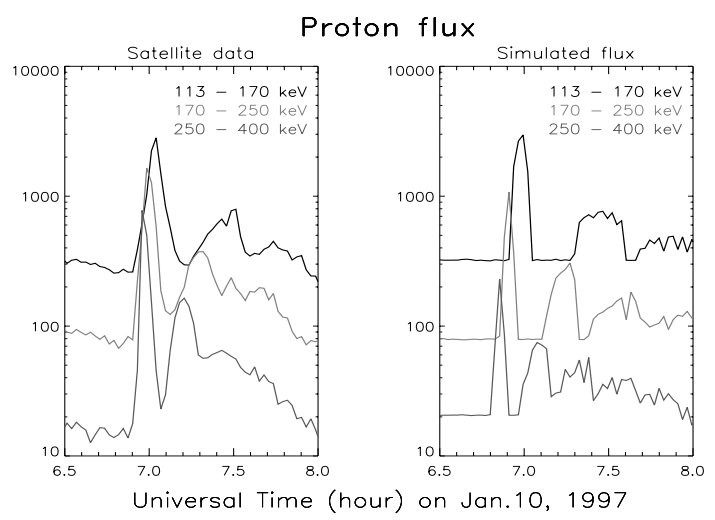

FIG. 12. Observed and calculated proton fluxes at geosynchronous orbit for a pulse with velocity $100 \mathrm{~km} / \mathrm{s}$; all other parameters are the same as in Fig. 11, except that for this case $\mathcal{E}_{0}=3.5 \mathrm{mV} / \mathrm{m} \cdot 9 \mathrm{R}_{\mathrm{E}}$

the other parameters unchanged) can sweep particles from about $12.3 \mathrm{R}_{\mathrm{E}}$ to geosynchronous orbit. For the maximum initial energy of about $16 \mathrm{keV}$, betatron acceleration will energize such a particle to $105 \mathrm{keV}$ at geosynchronous orbit. These extreme field values however are not expected to appear during many substorms, which is consistent with the observations in [2], where it is noticed that such very high energy injections occur in less than $10 \%$ of the total number of substorms surveyed. However, in some substorm events [8] the E-field can be higher $(30 \mathrm{mV} / \mathrm{m})$ at about $15 \mathrm{R}_{\mathrm{E}}$, which corresponds to $50 \mathrm{mV} / \mathrm{m}$ at $9 \mathrm{R}_{\mathrm{E}}$ in our model, due to the $1 / r$ dependence, and also $V_{0}$ can be much higher $(500 \mathrm{~km} / \mathrm{s})$. A pulse with these parameters can energize particles to much higher energies and may be responsible for the $10 \%$ of the cases mentioned. Intermediate values of the electric field $(15 \mathrm{mV} / \mathrm{m}$ at $15 \mathrm{R}_{\mathrm{E}}$ ) have also been reported [7] and can also be responsible for higher energization than presented in this example.

It is to be noted that the pulse parameters we chose above are not the only ones to give flux levels consistent with observations. Observations show that there is a wide range for the values of the electric field observed at the time of substorms as well as the plasma Earthward flow speed. Thus, it is instructive to show how a pulse with lower velocity and electric field can affect the proton flux. We choose both $V_{0}$ and $\mathcal{E}_{0}$ to be close to one half 
of the values in the previous simulation case $\left(V_{0}=100 \mathrm{~km} / \mathrm{s}\right.$, which is also chosen in [1] in their simulation, and $\mathcal{E}_{0}=3.5 \mathrm{mV} / \mathrm{m} \cdot 9 \mathrm{R}_{\mathrm{E}}$ ) with all other pulse parameters taken to be the same. The simulated proton fluxes are shown in Fig. 12 and are very similar to the previous case. All energized particles in this case also arrive from distances less than $9 \mathrm{R}_{\mathrm{E}}$. The small difference in the flux enhancement between these two cases is mainly due to the different particle azimuthal motion. With a lower propagating velocity in the second case, more particles will enter the pulse azimuthally in the radial distance of $6.6-9 \mathrm{R}_{\mathrm{E}}$. However, because more particles also exit the pulse azimuthally, on average the number of particles staying in all of the pulse is about the same. Thus, as can be seen in Fig. 12, the obtained flux levels in the second case are very close to those obtained in the first case.

\section{B. Electron injection event}

While our calculations have been performed for non-relativistic particles, our orbit solutions for electrons with the energy range of $100-300 \mathrm{keV}$ are good approximations to the exact relativistic ones. Thus, we look at the first electron injection event on January 10, 1997 (see Fig. 13), which is the one studied in [1] in their particle simulation model. We note that while their model uses a plane-wave E-field in Cartesian coordinates, in our model we consider that the geometry of the problem requires a cylindrical wave form (with a field of the form $E_{\phi}\left(r+V_{0} t, \phi\right)=-\left(\mathcal{E}_{0} / r\right) \mathcal{R}\left(r+V_{0} t\right) \Phi(\phi)$, as given by Eq. (10)), because a large portion of the particle trajectory takes place at distances from Earth less than $9 \mathrm{R}_{\mathrm{E}}$, where the background field has a significant spherical symmetry. A plane-wave solution is certainly not suitable in such a geometry for pulses of large azimuthal extent, and in general a wave solution with constant $V_{0}$ and an E-field not varying along the radial direction of

propagation will not conserve the field energy in a cylindrical geometry because the field energy density would be the same in such a wave, but the volume that the wave energy is confined decreases as the wave propagates towards lower $r$.

The pulse parameters for this electron injection event are: $\mathcal{E}_{0}=4.4 \mathrm{mV} / \mathrm{m} \cdot 9 \mathrm{R}_{\mathrm{E}}, V_{0}=$ 

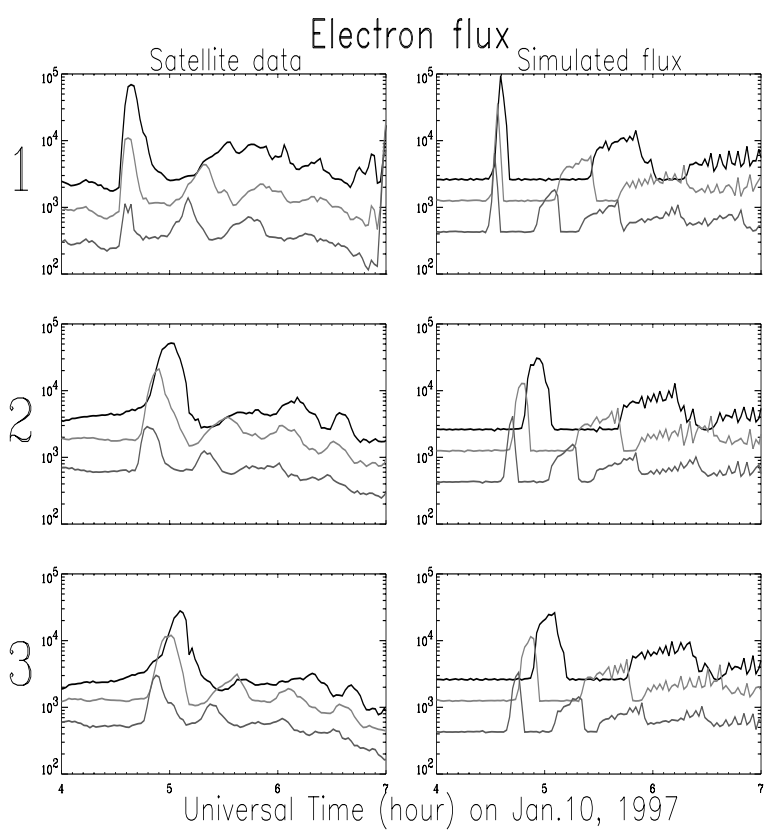

FIG. 13. Electron flux in the vicinity of $6.6 \mathrm{R}_{\mathrm{E}}$ as measured by three LANL satellites (left panel) and simulated at that satellite position (the graphs on the right); numbers 1, 2 and 3 correspond to satellites 1990-095 (LT $=\mathrm{UT}-2: 30), 1991-080(\mathrm{LT}=\mathrm{UT}+4: 42)$ and 1994-084 $(\mathrm{LT}=\mathrm{UT}+$ 6:54), respectively. The three energy ranges, shown in different shades of grey, are, from top to bottom in each graph: $[105,150],[150-225],[225-315] \mathrm{keV}$.

$100 \mathrm{~km} / \mathrm{s}, \Delta_{1}=\Delta_{2}=\Delta_{3}=11,000 \mathrm{~km}$, and $2 \delta \phi=30^{\circ}$. The background magnetic field parameter is chosen as $\mathrm{B}_{\mathrm{E}}=0.3 \mathrm{G}$ which corresponds to similar average background field at geosynchronous orbit as that used in [1]. The electron distribution parameters are taken to be $\kappa=4$ and $\varepsilon=2 \mathrm{keV}$, which is larger than the value used in [1], because our region of interest is closer to Earth than the one in their model. To model the particle loss after injection by the pulse we use a decay factor having an e-folding time of 3 hours, which is the value used in [1] for the same event. With these parameters the largest distance electrons are injected from is $8.9 \mathrm{R}_{\mathrm{E}}$, corresponding to those electrons that enter and exit the pulse radially. These electrons will experience an energy increase of 2.45 times via betatron acceleration (actually a little less, due to relativistic corrections). Other electrons will arrive at $6.6 \mathrm{R}_{\mathrm{E}}$ from distances closer to the Earth and will be less energized but will account for the initial flux increase. 
Fig. 13 shows the observed and simulated fluxes at the geosynchronous orbit for three satellites. The first satellite (1990-095) is near midnight (at about 2:00 local time) at the time of the injection, and thus the first peak at its position is quite narrow. The other 2 satellites are located eastward relative to the first one, and so there is a larger dispersion seen at these locations, due to the different azimuthal drifts for different electron energies. In all graphs, the simulated flux peaks are narrower than the observed ones as in the ion injection case and this is due to the reasons already presented.

A more realistic model would have to consider the possibility of a small number of particles being injected at azimuths outside the finite azimuthal extent of the pulse considered here. Also, the higher energy particles arriving earlier at the satellite location may energize particles in the local population there (perhaps by the instabilities they drive) thus the observed fluxes will be wider than our calculated ones. Another possible improvement is to include relativistic effects on the electron calculation in these energy ranges. For relativistic particles, the radial equation of motion is the same as for non-relativistic ones (the $\mathbf{E} \times \mathbf{B}$ is the same), so most of the particles (the ones that enter and exit the pulse radially) will come from the same distance as in the non-relativistic case. A simple calculation shows that the energization our highest energy electrons $\left(W_{f}=315 \mathrm{keV}\right.$, so $\left.\gamma_{f}=1.6\right)$ undergo is lowered from the calculated non-relativistic value of 2.45 to 2.2 , and so the change in the initial distance (to obtain the same energization as before) is less than $0.5 \mathrm{R}_{\mathrm{E}}$.

In conclusion, one sees that while all those effects mentioned would provide a "smoother" flux, they will not have a large effect on the peak flux values, and our model gives a good approximation for the injected flux levels.

It is to be noted that this energetic electron flux enhancement event has also been modeled by test particle simulations [6,1] with a good agreement with observation. However, there is a clear difference between our model and the test particle simulations in [1]. In our model we use an electric field pulse with a $1 / r$ factor multiplying a constant radial form in the pulse propagating frame so that the peak electric field amplitude increases as the pulse propagates toward the Earth. In the simulation study in [1] a much more complicated electric field wave 
form with a peak amplitude constant in time was used. In our model we find that the initial radial distance from which the particles are swept to the geosynchronous orbit is at around $8-9 R_{E}$ and thus the flux enhancement observed at geosynchronous orbit is due to electrons with an initial distance between $6.6 \mathrm{R}_{\mathrm{E}}$ and $9 \mathrm{R}_{\mathrm{E}}$. On the contrary, the simulation results in [1] indicate that more than $90 \%$ of the energetic electron flux enhancement is due to electrons with an initial distance $\geq 9 \mathrm{R}_{\mathrm{E}}$.

\section{SUMMARY AND CONCLUSIONS}

Energetic particle flux enhancement events in a wide range of magnetospheric disturbances can be modeled by considering particle interaction with an Earthward-propagating pulse with consistent $\mathbf{E}$ (westward directed) and $\mathbf{B}$ fields. Particle interaction with this pulse can account for the observed "dispersionless injection" events associated with these disturbances. As particles are swept Earthward by the pulse via $\mathbf{E} \times \mathbf{B}$ drift motion to a higher magnetic field location, their energy will be greatly increased due to the conservation of magnetic moment. Depending on the azimuthal extent of the pulse and the magnetic moment of the particles, particle azimuthal drift motion determines how long particles can stay in the pulse and thus their final position and energy gain. Analytical orbit solutions

for non-relativistic particles interacting with a cylindrical-wave pulse of a trapezoidal radial field profile and a finite azimuthal extent has been obtained. The model was then applied to energetic particle flux enhancement events associated with substorms, and the simulated particle fluxes are in good agreement with satellite observations.

Several general features of the particle orbit and energization can be drawn from this study. First, the particle energy gain depends only on the initial and final values of the magnetic field at the particle positions. Particles entering and exiting the pulse radially will be swept the longest distance and their energy gain will be the largest. Second, above a critical energy (which depends on the pulse parameters) particles can not stay in all three parts of the pulse and will exit the pulse azimuthally and they will not be as highly ener- 
gized. These particles azimuthally exiting the pulse at the observing satellite radial distance contribute to the initial phase of the flux enhancement, but have a small contribution to the overall observed flux level. Third, particles swept to the final location $\left(r_{f}, \phi_{f}, t_{f}\right)$ come from a larger initial radial distance at $\left(r_{i}, \phi_{i}, t_{i}\right)$, which is determined by the pulse parameters and the particle magnetic moment; in general, $r_{i}$ will be larger if the pulse propagating speed $V_{0}$ is lower and the radial pulse length is larger because particles will stay longer in the pulse. Also, $r_{i}$ will be larger if the electric field amplitude is larger because the larger $\mathbf{E} \times \mathbf{B}$ drift will be closer in magnitude to $V_{0}$ thus allowing the particles to remain in the pulse longer.

A great advantage of our model is that analytical particle orbit solutions are obtained and provide valuable physical insights into the problem and greatly reduce the amount of numerical computation needed for obtaining the injected fluxes at the observing satellite location. The approach of our model is also general in the sense that any radial wave form can be approximated by linear segments that we considered, and analytical orbit solutions are available. Thus, the injected fluxes can be easily obtained numerically by decomposing the pulse into a number of linear segments depending on the required accuracy for approximating the pulse form.

The good agreement between our simulated particle fluxes and the observed fluxes as well as other comparison studies of test particle simulations $[6,1]$ indicate that dispersionless energetic particle flux enhancement can be modeled by an Earthward propagating pulse with a westward electric field. Thus, more complex models such as the injection boundary model [16] are not necessarily needed in order to explain the dispersionless particle injection events. However, there is a clear difference between the results of our model and the results of test particle simulations in [1]. From our model we obtain the initial radial locations from which the particles are injected to the geosynchronous orbit to be at around $8-9 R_{E}$, which is much closer to the Earth than those obtained from the simulations in [1], who conclude that more than $90 \%$ of the energetic electron enhancement is due to electrons with an initial distance $\geq 9 \mathrm{R}_{\mathrm{E}}$. 


\section{ACKNOWLEDGMENTS}

This work was supported by the NSF Grant No. ATM-9523331 and the DoE Contract No. DE-AC02-76-CHO3073. We would like to thank R. Belian and G. Reeves for providing the LANL satellite data. 


\section{REFERENCES}

[1] X. Li et al., Geophys. Res. Lett. 25, 3763 (1998).

[2] R. Belian, D. Baker, P. Higbie, and E. Hones, J. Geophys. Res. 83, 4857 (1978).

[3] T. Moore, R. Arnoldy, J. Feynman, and D. Hardy, J. Geophys. Res. 86, 6713 (1981).

[4] L. Lanzerotti, C. Roberts, and W. Brown, J. Geophys. Res. 72, 5893 (1967).

[5] F. Bogott and F. Mozer, J. Geophys. Res. 79, 1825 (1974).

[6] X. Li et al., Geophys. Res. Lett. 20, 2423 (1993).

[7] G. Sheperd et al., J. Geophys. Res. 85, 4587 (1980).

[8] T. Aggson, J. Heppner, and N. Maynard, J. Geophys. Res. 88, 3981 (1983).

[9] T. Northrop, The Adiabatic Motion of Charged Particles (Interscience Publishers, New York, 1963), p. 12.

[10] J. Roederer, Dynamics of Geomagnetically Trapped Radiation (Springer-Verlag, Berlin, 1970), p. 87.

[11] L. Lyons and D. Williams, Quantitative Aspects of Magnetospheric Physics (D. Reidel, Boston, 1984), p. 20.

[12] V. Vasyliunas, J. Geophys. Res. 73, 2839 (1968).

[13] S. Christon et al., J. Geophys. Res. 96, 1 (1991).

[14] J. Birn et al., J. Geophys. Res. 102, 2325 (1997).

[15] M. Schultz and L. Lanzerotti, Particle Diffusion in the Radiation Belts (Springer-Verlag, Berlin, 1974).

[16] C. McIlwain, in Magnetospheric Physics, edited by B. McCormac (D. Reidel, Hingham, Mass., 1974), p. 143. 
[17] T. Moore, D. Gallagher, J. Horwitz, and R. Comfort, Geophys. Res. Lett. 14, 1007 (1987).

[18] M. Hudson et al., Geophys. Res. Lett. 22, 291 (1995). 